\title{
Determining Shock and Detonation Wave Propagation Time Using Wavelet Methods
}

\author{
Frank K. Lu, and A. Albert Ortiz ${ }^{\dagger}$ \\ Aerodynamics Research Center, University of Texas at Arlington, Arlington, TX 76019, USA
}

The propagation speed of a shock or detonation wave in a shock or detonation tube is usually determined by a time-of-flight method by dividing the distance between two transducers with the propagation time of the disturbance signal. Some arbitrariness is inherent in determining the propagation time by this method. A new method based on Haar and Morlet wavelet transforms is reported. The method was tested against a step and a decaying spike with added noise, which are representative of shock and detonation waves. The wavelet methods can be applied to the step discontinuity provided that the SNR ratio is good. The wavelet methods worked very well for the decaying spike in the presence of noise.

\section{Nomenclature}

$E[\quad$ Expected value of

$f_{s} \quad$ Sampling frequency

$n_{d} \quad$ Number of averages

$N \quad$ Sample size

TOF Time-of-flight

WCCC Wavelet cross-correlation coefficient

WECC Wavelet envelope correlation coefficient

$\bar{u} \quad$ Average wave propagation speed

$\Delta L \quad$ Distance between two transducers

$\Delta t \quad$ Time-of-flight of a disturbance between two sensors; also, sampling period

$\theta_{x y} \quad$ Phase angle

$\tau_{x y} \quad$ Time delay

$\sigma \quad$ Standard deviation

$\psi \quad$ Wavelet function (mother wavelet)

$\psi_{a, b} \quad$ Translated and dilated wavelet function (daughter wavelet)

$\Psi \quad$ Fourier transform of wavelet function

$<>\quad$ Mean ensemble value

() Estimate

${ }^{*}$ Professor, Department of Mechanical and Aerospace Engineering, Associate Fellow AIAA.

$\dagger$ Graduate Research Assistant, Department of Mechanical and Aerospace Engineering.

Copyright (c) 2009 by F.K. Lu and A.A. Ortiz. Published by the American Institute of Aeronautics and Astronautics, Inc. with permission. 


\section{Introduction}

$\mathrm{T}$

The determination of the propagation speed of a shock or detonation wave as in shock or detonation tube experiments typically makes use of the time-of-flight (TOF) method. In this method, the speed of a propagating shock, say, is obtained by determining the passage time of the wave $\Delta T$ between two transducers with a known separation distance $\Delta L$, to yield

$$
\widehat{u}_{s}=\Delta L / \Delta T .
$$

Some recent examples of the TOF method for shock and detonation wave studies include Refs. [1-3] and for other propagating signals include Refs. $[4,5]$. The TOF method appears to be suitable for strong, distinct transient signals such as shock and detonation waves. In situations where the signal is weak, the TOF method requires further treatment or may even have to be replaced by more powerful methods.

Moreover, even though the TOF method is valid in principle and is simple to apply to propagating shock or detonation waves, its practical implementation can be subjective. This is because the shock front is not measured as a step rise, as in theory, but is spread over a small time interval. This interval depends on a number of factors, such as the size of the transducer, viscous effects and the data sampling rate. Different arbitrary criteria can thus be formulated for determining the shock arrival over a transducer. For example, the shock may be deemed to have arrived at the transducer when the signal rises above an arbitrary threshold, or when the signal is midway between arbitrarily defined upstream and downstream values. These criteria, which yield signal arrival times close to one another, are subjective. They may be unreliable if the error of the time estimate is comparable to $\Delta T$. Such a situation may occur if the transducers are closely spaced or if the sampling rate is low. For the same record length, increasing the sampling rate does not fundamentally overcome this difficulty although it may reduce the uncertainty in the $\Delta T$ estimate.

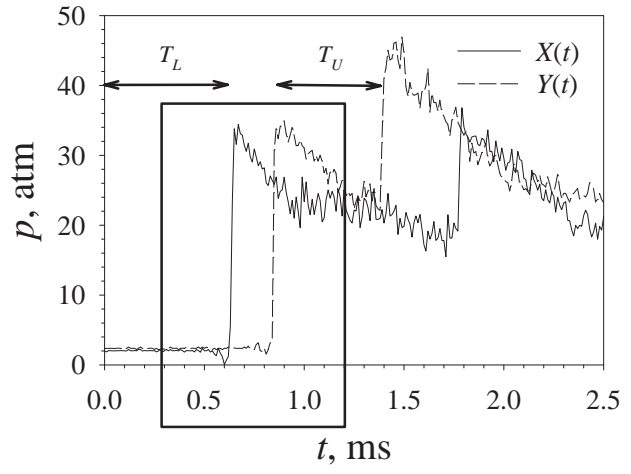

(a) Detonation wave propagating past two transducers.

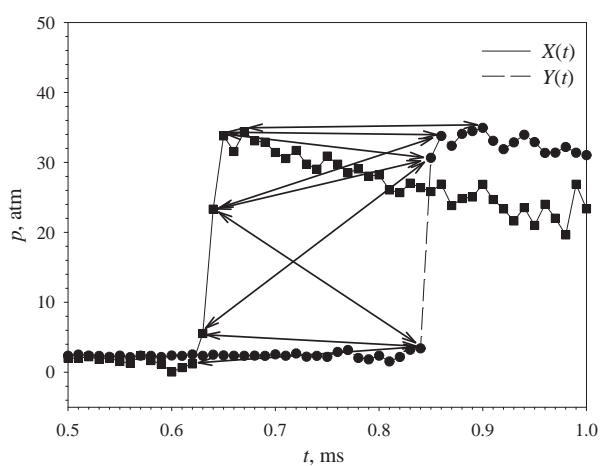

(b) Enlargement of wave fronts.

Figure 1. Arbitrariness of the TOF method.

To illustrate this arbitrariness, consider the propagation of a fully-developed Chapman-Jouguet (CJ) detonation wave past two transducers in a stoichiometric oxyhydrogen mixture initially at $2.01 \mathrm{~atm}$, Fig. 1, the data being reported previously in Ref. [6]. (Figure 1(a) includes other features, including a subsequent wave reflection, that will be described later.) The region where the pressure initially rises is enlarged and shown in Fig. 1(b). The symbols represent actual data, sampled at $100 \mathrm{kHz}$ per channel with a simultaneous sample-and-hold system. Figure 1(b) shows that the two wave fronts $X(t)$ and $Y(t)$ are spread over a few 
data points. The double-headed arrows indicate possible pairs of data points for the TOF method, resulting in estimates of $\Delta T$ from 0.21 to $0.23 \mathrm{~ms}$. The $0.02 \mathrm{~ms}$ uncertainty represents a ten percent error on the estimate of the velocity.

Previous work ${ }^{6,7}$ examined the use of statistical methods for estimating the time-of-flight of shock and detonation waves. These transient events do not easily lend themselves to the usual time- and frequencydomain analysis which assumes stationarity and ergodicity. ${ }^{8,9}$ These assumptions are very powerful in allowing large amounts of data to be processed to obtain statistical measures. Unfortunately, the transient nature of propagating events limits the amount of data available. Here, a wavelet approach is introduced for estimating the time-of-flight.

\section{Background on Wavelet Transforms}

Before discussing the techniques that were developed, a brief background on wavelet transforms is provided. An advantage of wavelet transforms is the ability to localize both frequency and time for a given signal. The localization of the time and frequency is the identification of the intervals in the time-frequency representation that contain the energy of the signal. ${ }^{10}$ The localization of both frequency and time with the Fourier method is problematic due to the fixed window size. ${ }^{10}$ In Fourier transforms, good frequency localization is achieved with a large window that indicates good frequency resolution but with poor time resolution throughout. However, good time localization is achieved with a small window that has good time resolution but poor frequency resolution. In other words, the Fourier transform can achieve good frequency or time localization by spreading the energy in the time or frequency domain. The wavelet transform resolves the issue of good frequency and time localization by having the width of the window vary from low to high frequency. The varying window allows the wavelet transform to achieve good time localization for high frequency bands, which tends to have a shorter time duration. Whereas for low frequency bands that are usually of longer time duration, the wavelet transform has good frequency localization. The wavelet transform has become particular useful in analyzing nonstationary signals due to the inherent properties associated with the transformation.

In fact, there are many types of time-frequency representations, such as wavelets, short-time Fourier transforms, and the Wigner-Ville distribution. ${ }^{11}$ All the time-frequency representations are governed by the time-frequency uncertainty principle that results in a tradeoff of the localization of time for frequency and vice versa. ${ }^{12}$ The uncertainty principle is a modification of the Heisenberg uncertainty principle applied for signal processing. The uncertainty principle is defined by the time-frequency bandwidth product

$$
\Delta_{t} \Delta_{\omega} \geq \frac{1}{2}
$$

which yields a lower bound for the time and frequency localization. The time-frequency bandwidth can be derived from the application of Schwartz' inequality to the time duration

$$
\Delta_{t}^{2}=\frac{1}{E} \int_{-\infty}^{\infty}(t-\langle t\rangle)^{2}|x(t)|^{2} d t=\int_{-\infty}^{\infty} t^{2} \frac{|x(t)|^{2}}{E} d t-\langle t\rangle^{2}
$$

and the frequency bandwidth

$$
\Delta_{\omega}^{2}=\frac{1}{2 \pi} \int_{-\infty}^{\infty}(\omega-\langle\omega\rangle)^{2} \frac{|X(\omega)|^{2}}{E} d \omega=\frac{1}{2 \pi} \int_{-\infty}^{\infty} \omega^{2} \frac{|X(\omega)|^{2}}{E} d \omega-\langle\omega\rangle^{2}
$$

which are both normalized by the energy of the signal. The derivation for the uncertainty principle is provided in several introductory wavelet texts. ${ }^{10,13,14}$ 


\section{II.A. Continuous Wavelet Transform}

The continuous wavelet transform (CWT) is the continuous-time transformation for a given signal. The CWT consists of a family of dilated and translated wavelets that is defined by

$$
\psi_{a, b}(t) \triangleq \frac{1}{\sqrt{|a|}} \psi\left(\frac{t-b}{a}\right)
$$

The mother wavelet is the template basis function that is used for determing the family of wavelets. The mother wavelet has to have a zero mean, which follows from the admissibility condition

$$
C_{\psi}=\int_{-\infty}^{\infty} \frac{|\Psi(\omega)|^{2}}{\omega} d \omega<\infty
$$

The admissibility condition is required if perfect reconstruction of the signal is needed. In most cases, the admissibility condition is satisfied in construction of the mother wavelet.

The mother wavelet is used to generate a family of daughter wavelets that have been dilated and translated with Eq. (5). Dilation of the wavelets is defined by scales, which are related to frequency. The scaling of the wavelet has a compression effect on the mother wavelet that is used to form a daughter wavelet. The compressed versions of the mother wavelet also have shorter time durations that allow for accurate time localization of the higher frequency component. The translation of the mother wavelet has no effect on the wavelet function other than a shift in time. An example of the Haar mother wavelet translated and dilated is shown in Fig. 2. Referring to Fig. 2, the top half shows the Haar

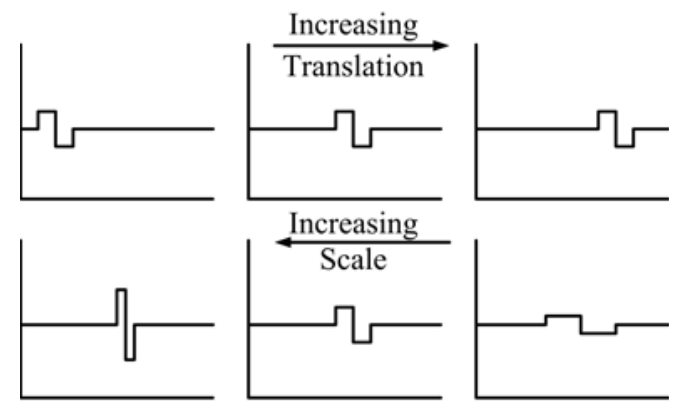
wavelet translated in time, which remains a uniform

Figure 2. A translation and dilation of the Haar wavelet.

shape throughout. The bottom half consists of the Haar wavelet located at the same time, but for various scales. The amplitude and duration vary with the scaling factor. With increasing scale, the amplitude decreases while the duration increases. The total energy is conserved throughout the various translations and dilations.

The family of daughter wavelets is orthogonal to each other and is used to compute the continuous wavelet transform $(\mathrm{CWT})$

$$
\operatorname{CWT}(a, b)=\int_{-\infty}^{\infty} \psi_{a, b}^{*}(t) f(t) d t
$$

The CWT has a varying scale and time grid that forms a constant relative bandwidth grid $Q$, defined by

$$
Q \triangleq 2 \Delta_{\omega} / \omega_{0}
$$

This constant relative bandwidth grid relates the spectral width to a mean frequency. An example of the constant relative bandwidth grid is shown in

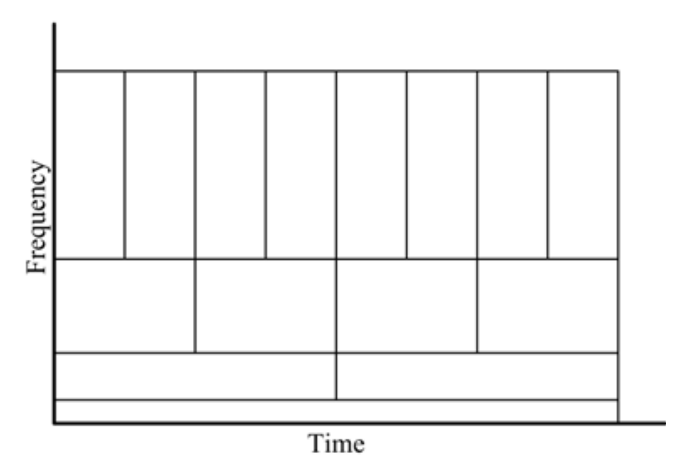

Figure 3. A general grid representing the varying time and frequency resolution for a CWT.

Fig. 3. The grid shows a finer frequency band but with poor time localization at low frequencies (i.e., large 
scales). On the other hand, the high frequencies (low scales) have a poor frequency band, but have better time localization. The CWT also conserves energy after the transformation justas in the Fourier transform.

Just as for the continuous Fourier transform, the CWT cannot be implemented in digital signal processing. A solution to this problem is to discretize the CWT. Note: this is not the same as the discrete wavelet transform (DWT), which applies high- and low-pass filters to determine the wavelet coefficients at various levels. A scalogram is used for displaying the CWT for various scales with time. The scalogram is a time-frequency representation commonly used for wavelet analysis. The scalogram is a representation of the energy spectrum, which is

$$
E_{C W T}(a, b)=|\operatorname{CWT}(a, b)|^{2}
$$

The wavelet phase angle is

$$
\theta_{C W T}(a, b)=\tan ^{-1}\left[\frac{\Im[C W T(a, b)]}{\Re[C W T(a, b)]}\right]
$$

and is neglected in some cases when the basis function consists of only real values. Another useful parameter is the wavelet ridge that is defined by

$$
\frac{d E_{C W T}(a, b)}{d a} \triangleq 0
$$

which is a representation of the local maxima of the energy spectrum. The wavelet ridge is particularly useful for extracting the extreme energy signatures from the energy spectrum.

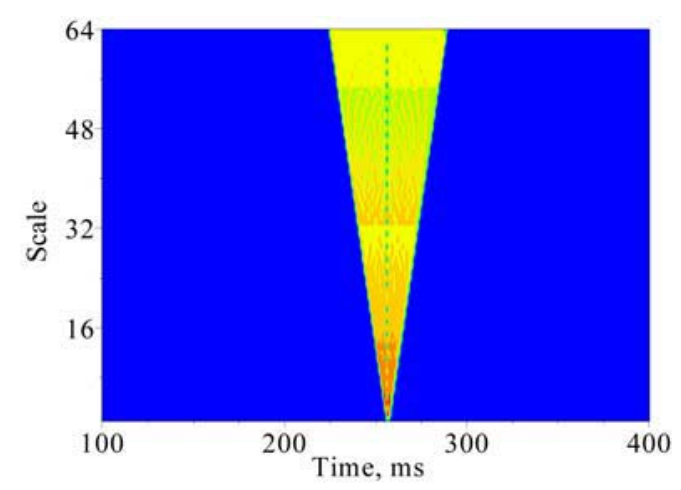

Figure 4. The effect of the cone of influence for an impulse function.

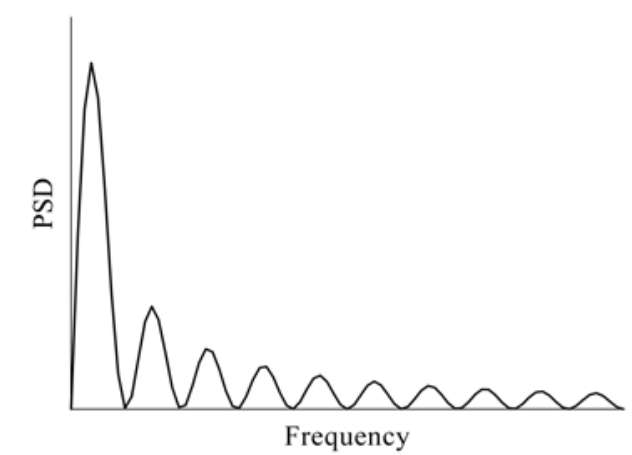

Figure 5. The frequency content for the Haar wavelet.

\section{II.B. Cone of Influence}

The cone of influence (COI) is the effect that discontinuities and the edges of a finite signal create in the scalogram. ${ }^{15}$ The COI can be considered to be a contamination of the spectrum, which increases linearly with increasing scale. ${ }^{15}$ An example of the COI for a Kronecker delta utilizing a Haar wavelet is shown in Fig. 4. Note that for the Haar wavelet, the width of the COI increases with a 1:1 ratio with scale. It can also be noted that this wavelet has good time localization of the discontinuity at low scale values or, equivalently, high frequencies.

\section{II.C. Selected Wavelets}

A brief discussion of the types of wavelets used in this study is now provided.

\section{II.C.1. Haar Wavelet}

The Haar wavelet is the first and simplest wavelet. It is also the least computational intensive wavelet and may be preferred for quick computations. The Haar wavelet is given by

$$
\psi(t) \triangleq\left\{\begin{array}{cc}
1 & 0 \leq t<\frac{1}{2} \\
-1 & \frac{1}{2} \leq t<1 \\
0 & \text { otherwise }
\end{array}\right.
$$


The Haar wavelet has good time localization but bad frequency localization. Referring to Fig. 5, the frequency information of the Haar wavelet has a broad peak with ripples throughout due to the discontinuity in the time domain. The applications for the Haar wavelet are limited due to the discontinuity.

\section{II.C.2. Morlet wavelet}

The Morlet wavelet is more complicated than the Haar wavelet. It is a modulated Gaussian function:

$$
\psi(t) \triangleq \frac{1}{\sqrt{\pi f_{b}}} e^{j 2 \pi f_{c} t} e^{-t^{2} / f_{b}}
$$

where

$$
f_{b}=\int f^{2} \Psi d f
$$

The Morlet wavelet is a complex wavelet function that does not satisfy the admissibility condition. ${ }^{12}$ Modifications to the Morlet wavelet can be done to satisfy the condition if reconstruction is necessary. The wavelet has good time and frequency localization since it has exponential decays in both domains. The localization is governed by the central frequency which, for high values, reduces the Morlet wavelet function to

$$
\psi(t)=\pi^{-1 / 4} e^{j 2 \pi f_{c} t} e^{-t^{2} / 2}
$$

The center frequency has a direct relationship on the number of oscillations that the wavelet exhibits. For high frequencies, the number of oscillations increases, improving the spectral resolution and decreasing the temporal resolution. ${ }^{16}$ An example of a Morlet wavelet in the time and frequency domain is provided in Fig. 6. Figure 6(a) shows the real and imaginary components of a Morlet wavelet function with a wavelet's central frequency of seven in the time domain. Figure 6(b) shows the Fourier transform of the Morlet wavelet which has a better frequency localization than the Haar wavelet shown in Fig. 5. See the appendix for further discussion of wavelet scales and the center frequency.

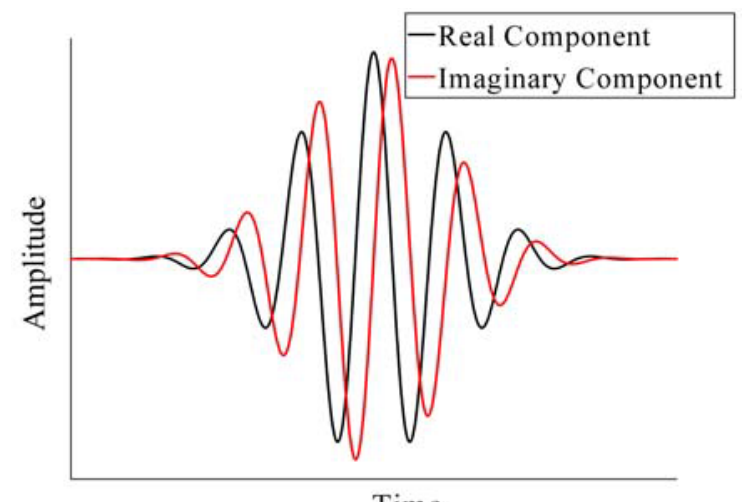

Time

(a) The real and imaginary components of the Morlet wavelet.

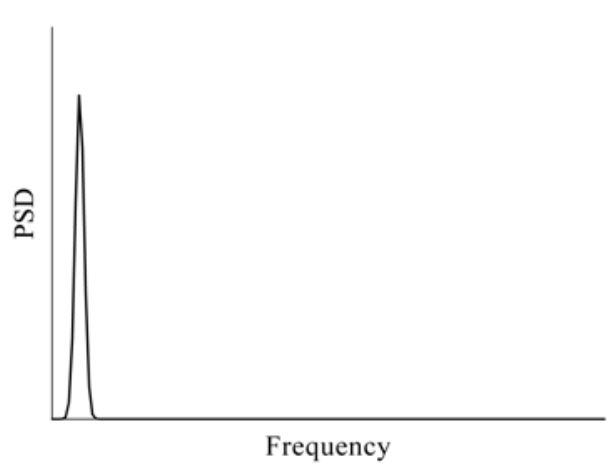

(b) Fourier transform of the Morlet wavelet.

Figure 6. The time and frequency domain for a Morlet wavelet.

\section{II.D. Wavelet Cross-Correlation Function}

A wavelet cross-correlation coefficient (WCCC) ${ }^{17}$ can be used to determine the correlation between the pair of signals in the frequency domain analogous to the nonstationary cross-spectral density phase method. ${ }^{7}$ 
However, the WCCC does not depend on the phase information for estimating the time delay. The WCCC makes use of the wavelet coefficients to determine the correlation between the pair of signals. The wavelet cross-correlation function is defined as

$$
\widehat{\mathrm{WC}}_{x y}(a, \tau) \triangleq \lim _{T \rightarrow \infty} \frac{1}{T} \int_{-T / 2}^{T / 2} \mathrm{CWT}_{x}^{*}(b, a) \mathrm{CWT}_{y}(b+\tau, a) d b
$$

from which one can define a wavelet cross-correlation coefficient

$$
\widehat{\mathrm{WR}}_{x y}(a, \tau) \triangleq \frac{\widehat{\mathrm{WC}}_{x y}(a, \tau)}{\sqrt{\widehat{\mathrm{WC}}_{x x}(a, 0) \widehat{\mathrm{WC}}_{y y}(a, 0)}}
$$

The WCCC has a range of $-1 \leq \widehat{\mathrm{WR}}_{x y} \leq 1$ where one signifies a strong correlation between the pair of signals. An unbiased WCCC function that corrects for edges of the window can be written in discrete form as

$$
\begin{aligned}
\widehat{\mathrm{WC}}_{x y}\left(a, i_{\tau}\right)= & \frac{1}{N-\left|i_{\tau}\right|} \sum_{i=0}^{N-1} \mathrm{CWT}_{x}^{*}(i, a) \mathrm{CWT}_{y}\left(i+i_{\tau}, a\right), \\
& i_{\tau}=-(N-1),-(N-2), \ldots, N-2, N-1
\end{aligned}
$$

The WCCC provided in Eq. (17) is a general equation for the correlation function. The WCCC has many variations depending on the mother wavelet used to transform the pair of signals. Each mother wavelet yields different wavelet coefficients that provide similar results, and may also provide added detail depending on how well the particular basis function models the signal.

\section{Results and Discussion}

To illustrate the above developments, the Haar and Morlet wavelets were applied to a number of model functions, similar to tests performed on the nonstationary cross-correlation and the nonstationary crossspectral density phase methods, ${ }^{6,7}$ namely:

- Unit step functions $X(t)$ and $Y(t)$ both with SNR $=8-32$ and with $Y(t)$ delayed by $\tau_{M}=50$;

- A unit step function $X(t)$ at $t=0$ with $\mathrm{SNR}=16$, and a step function $Y(t)$ at $\tau_{M}=50$ with an amplitude of $A=2^{-2}-2^{3}$ and $\mathrm{SNR}=16$

- Exponentially decaying functions $X(t)=e^{-b t / 100}$ and $Y(t)=e^{-b\left(t-\tau_{M}\right) / 100}$, where $b=2^{-2}-2^{2}$ and $\mathrm{SNR}=16$ for both functions, and $\tau_{M}=50$;

- Linearly decaying functions $X(t)=1-c t / 100$ and $Y(t)=1-c\left(t-\tau_{M}\right) / 100$, where $c=2^{-2}-2^{2}$ and $\mathrm{SNR}=16$ for both functions, and $\tau_{M}=50$.

Uniformly distributed white noise was added to the model functions. The SNR was determined by

$$
\mathrm{SNR}(\mathrm{dB})=10 \log _{10} P_{\text {signal }} / P_{\text {noise }}
$$

where $P_{\text {signal }}$ and $P_{\text {noise }}$ represent the power of the signal and noise respectively. For all the functions, the sample size was set to 1001 and the time step $\Delta t=1$. These functions are displayed in Fig. 7 . 


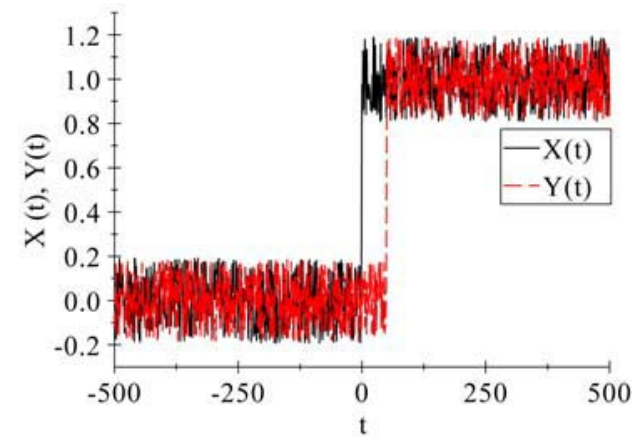

(a) Step function; various SNR.

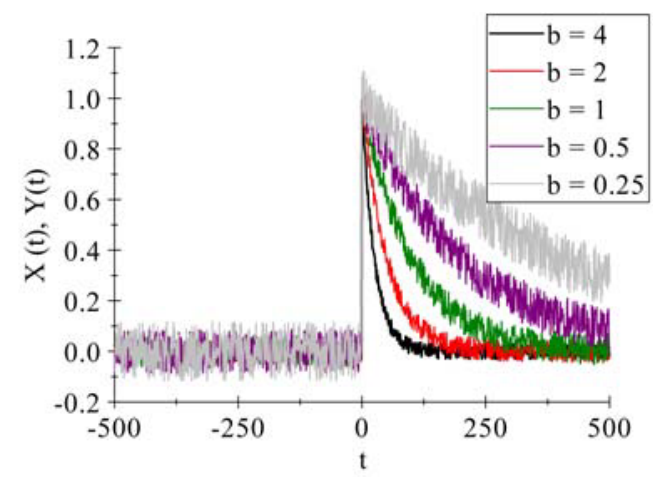

(c) Exponential decay with SNR $=16$ (only $X(t)$ is shown).

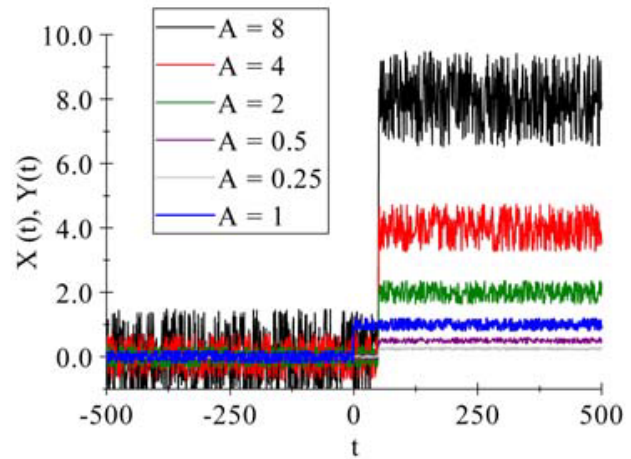

(b) Step function; various amplitudes for $Y(t)$, both with SNR $=16$.

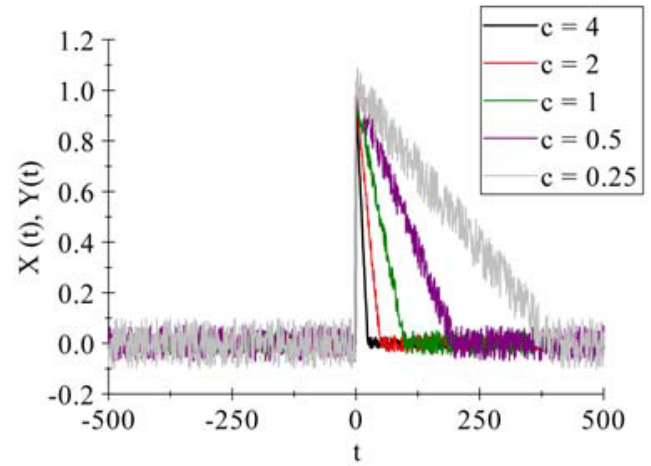

(d) Linear decay with SNR $=16$ (only $X(t)$ is shown).

Figure 7. Model functions. 


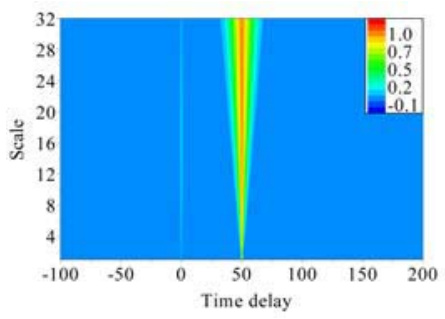

(a) $\mathrm{SNR}=32$.

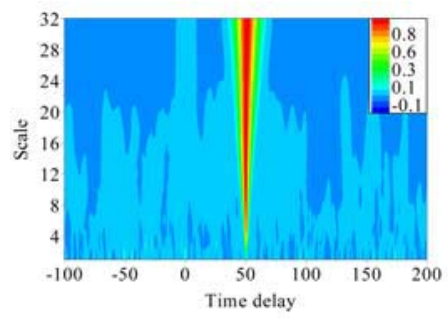

(b) SNR $=16$.

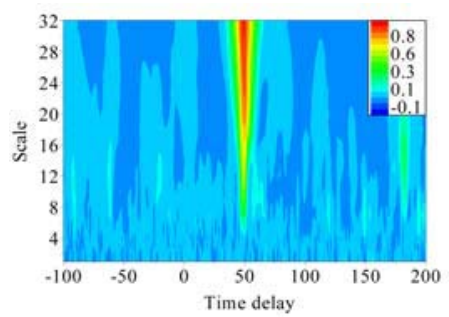

(c) $\mathrm{SNR}=8$.

Figure 8. WCCC Haar for step function with different SNR.

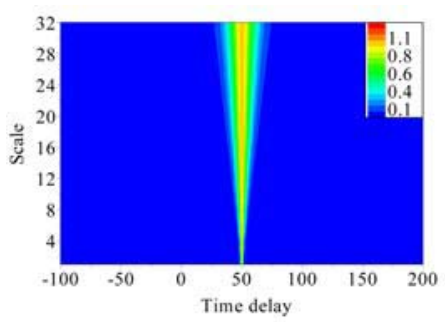

(a) $\mathrm{SNR}=32$.

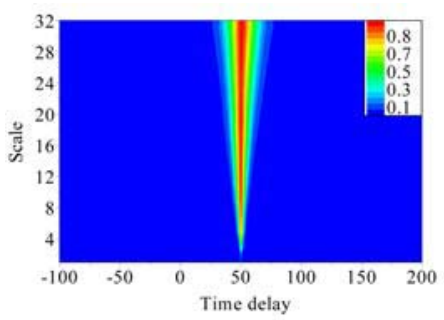

(b) SNR $=16$.

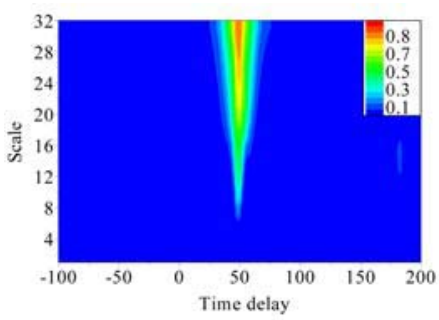

(c) $\mathrm{SNR}=8$.

Figure 9. WECC Haar for step function with different SNR.

\section{III.A. Step Change with Different Signal-to-Noise Ratio and Varying Amplitudes}

The results for this case with the WCCC-Haar and WECC-Haar methods are displayed in Figs. 8 and 9 respectively. The WCCC-Haar method was able to capture the correct time delay between the signals when the SNR was 16 and 32. For the case were the SNR was the poorest, namely 8, the low scale values of 1-5 (corresponding to high frequencies) were ignored as they differed significantly from the rest of the scales. For example, Fig. 8(a) shows a strong correlated peak at a time delay of 50 for all scales, which corresponds to the actual time delay. For SNR = 16, Fig. 8(b) shows a strong correlated peak that is smeared at around a time delay of 50 . The correlated peak weakens for low scale (i.e., high frequencies), but the peak is still stronger than the surrounding to provide the correct time delay for the signals. For SNR = 8, Fig. 8(c) shows a strong correlated peak that weakens significantly with lower scales. Strong noise appears to only be effective for the low scales (1-5), which corresponds to the values where the time delay is incorrect.

The results for the WECC-Haar method are generally identical to the WCCC-Haar method. However, for the SNR $=8$ case, Fig. 9(c) shows a broad correlated peak at large scales, which makes it difficult to graphically determine the time delay at the low scales (1-8). The numeric results are the same as for the WCCC-Haar method, and is unable to provide valid time delay results for the scales $1-5$.

The WCCC-Morlet was able to properly capture the time delay for large SNR but was unable to determine the time delay for poor SNR as can be seen in Fig. 10(a). When the SNR = 16, the correlation between the signals is weaker as it is more difficult to determine the time delay graphically. The correlated peak is generally around a delay of 50 except for the scales $16-18$ and 10-12. The correlated peak is also lost graphically for the low scales $1-4$, which correspond to erroneous time delay values. When the SNR $=8$, the WCCC-Morlet method is unable to determine the time delay as can be seen in Fig. 10(c). Results from 


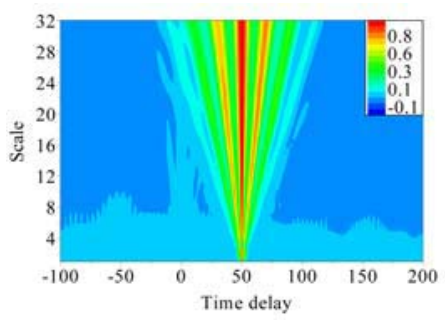

(a) $\mathrm{SNR}=32$.

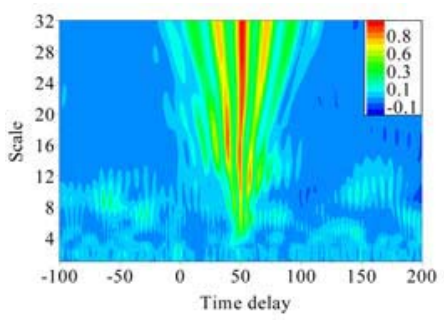

(b) SNR $=16$.

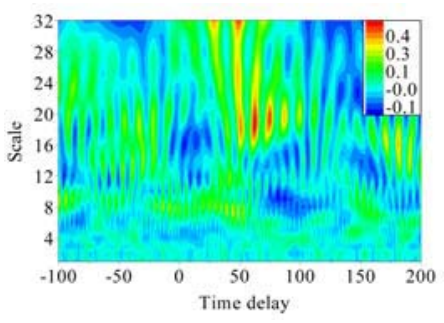

(c) $\mathrm{SNR}=8$.

Figure 10. WCCC Morlet for step function with different SNR.

the WECC-Morlet method are identical to the WCCC-Morlet method and the figures are not shown for brevity. The wavelet analysis for the case shown in Fig. 7(b) yields results similar to the above and are also not discussed for brevity.

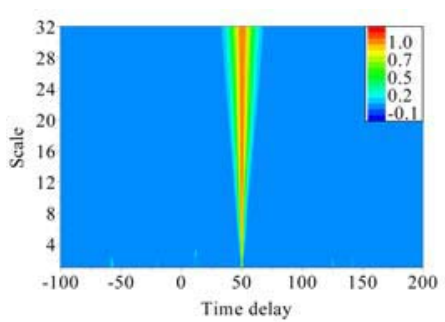

(a) $b=2^{-2}$.

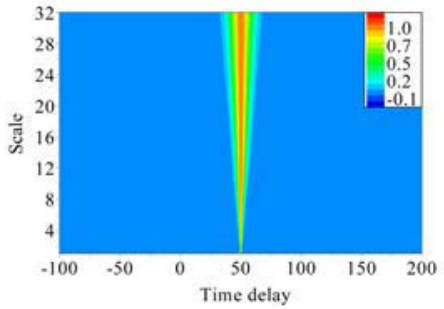

(b) $b=2^{-1}$.

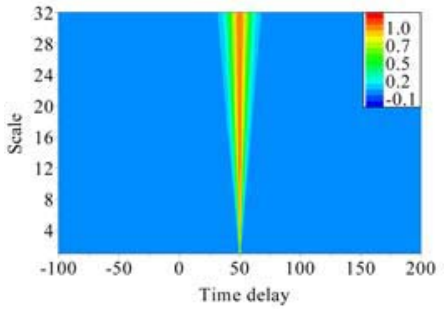

(c) $b=2$.

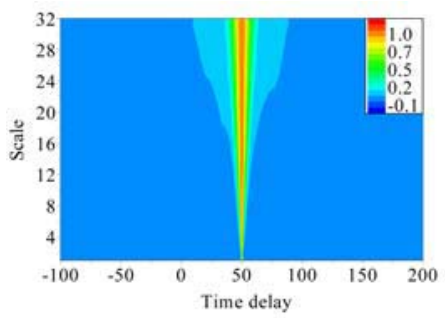

(d) $b=2^{2}$.

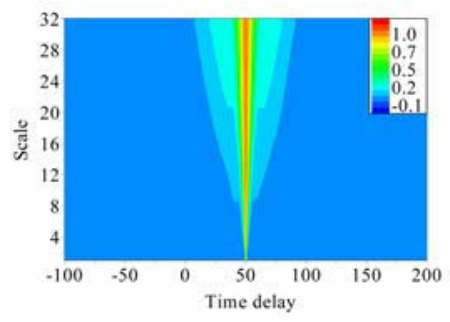

(e) $b=2^{4}$.

Figure 11. WCCC-Haar method for an exponentially decaying function.

\section{III.B. Exponential and Linear Decay}

The time delay results using the WCCC-Haar method for such a signal are very good compared to the step function discussed above; see Fig. 11. The results correctly identify the time delay with no deviation for all scales. The figures are similar to the WCCC-Haar SNR=16 result except the correlated band is less broad and the noise for the surrounding correlation function is minimized to have a smooth surface at time delay values away from the correlated peak. The different decay rates have a minimal effect in defining the correlation peak although the correlation peak becomes slightly sharper with a faster decay rate. The 


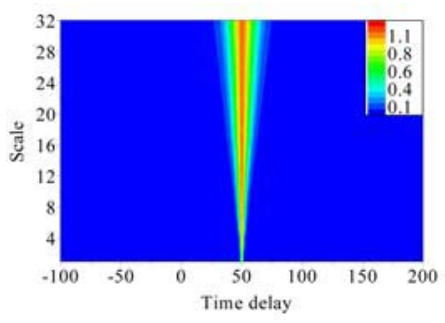

(a) $b=2^{-2}$.

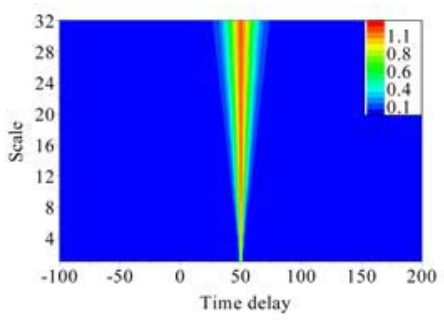

(b) $b=2^{-1}$.

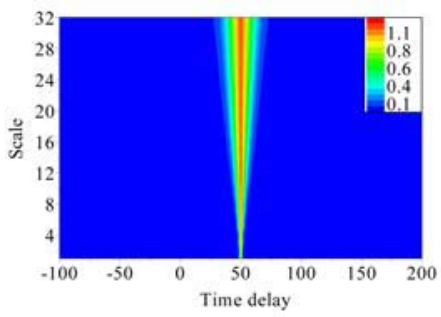

(c) $b=2$.

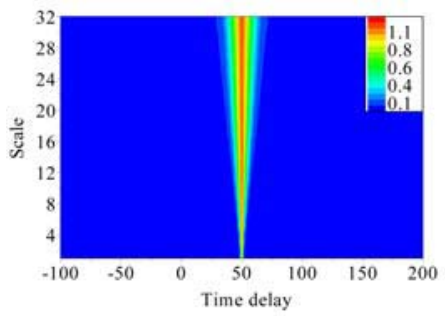

(d) $b=2^{2}$.

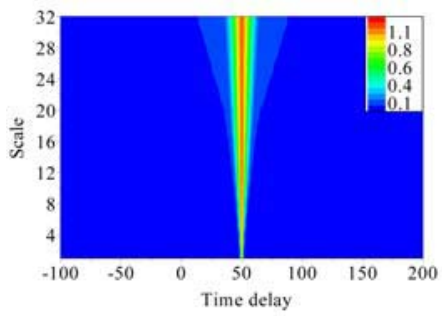

(e) $b=2^{4}$.

Figure 12. WECC-Haar method for an exponentially decaying function.

correlated peak influence also increases for a larger range of time delay values at larger scale values for faster decay rates.

The WECC-Haar method has similar results to the WCCC-Haar method and the results are shown in Fig. 12. The time delay is correctly identified with no variance. The only difference between the two Haar methods is that the correlated peaks are slightly sharper for the WECC-Haar than the WCCC-Haar method.

Turning to the WCCC-Morlet method, it also provides good time delay estimates for the decaying signals, see Fig. 13. The subfigures show a clear graphical correlation between the signals compared to results from the step functions for similar SNR. The correlated peak's influence becomes weaker for the slower decay rates, but are still strong enough to allow the time delay to be determined accurately. Finally, the WECCMorlet method yields an improvement over the WCCC-Morlet method due to the more defined correlation functions. All the time delay values are at 50 with zero variance. Finally, due to the similarity between the linear decay and the exponential decay, the results for the linear decay are not discussed for brevity.

\section{III.C. Summary of Results}

The ability of these wavelet methods to determine the time delay can be seen in Table 1 . The table shows that both the Haar and Morlet wavelets were not able to properly determine the time delay for a noisy step discontinuity. On the other hand, both wavelets with and without an envelope function, performed extremely well in estimate the time delay. More important perhaps is their ability to determine the variance of the estimate. 


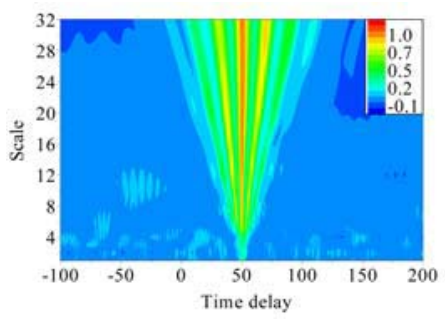

(a) $b=2^{-2}$.

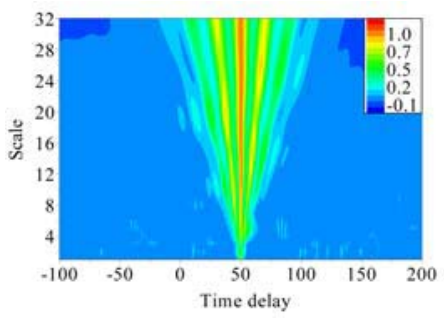

(b) $b=2^{-1}$.

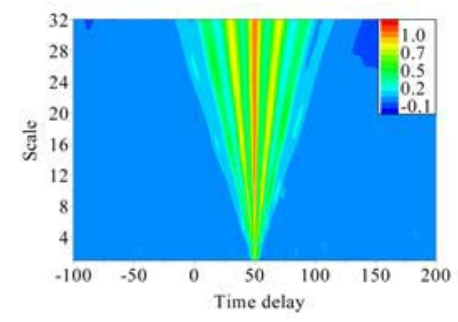

(c) $b=2$.

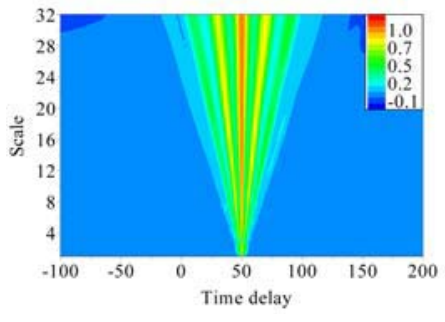

(d) $b=2^{2}$.

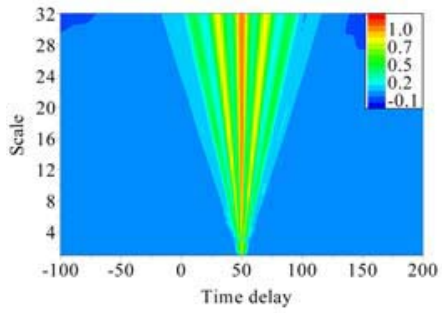

(e) $b=2^{4}$.

Figure 13. WCCC-Morlet method for an exponentially decaying function.

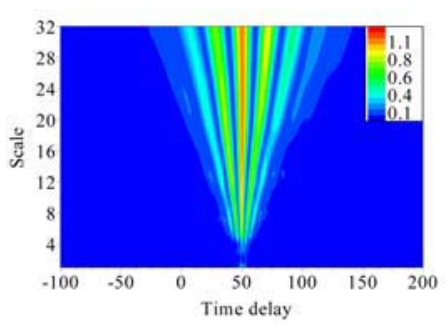

(a) $b=2^{-2}$.

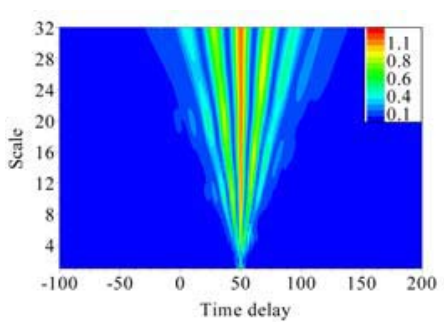

(b) $b=2^{-1}$.

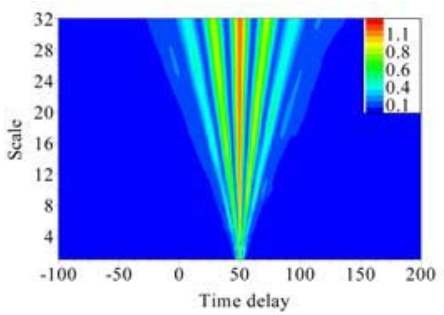

(c) $b=2$.

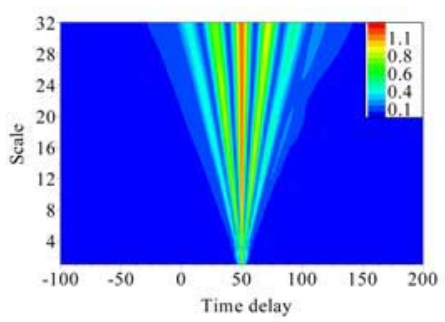

(d) $b=2^{2}$.

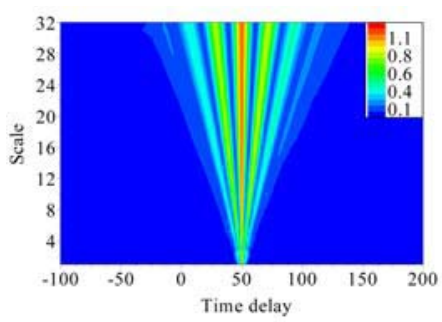

(e) $b=2^{4}$.

Figure 14. WECC-Morlet method for an exponentially decaying function. 
Table 1. WCCC and WECC time delay values for model functions of Fig. 7 using Haar and Morlet wavelet crosscorrelations with and without envelope

\begin{tabular}{|c|c|c|c|c|c|c|c|c|}
\hline & \multicolumn{2}{|c|}{ Haar } & \multicolumn{2}{|c|}{ Morlet } & \multicolumn{2}{|c|}{ Haar } & \multicolumn{2}{|c|}{ Morlet } \\
\hline & \multicolumn{8}{|c|}{ Step change with different signal-to-noise ratio } \\
\hline & \multicolumn{2}{|c|}{ WCCC } & \multicolumn{2}{|c|}{ WECC } & \multicolumn{2}{|c|}{ WCCC } & \multicolumn{2}{|c|}{ WECC } \\
\hline & $\mu_{t}$ & $\sigma_{t}$ & $\mu_{t}$ & $\sigma_{t}$ & $\mu_{t}$ & $\sigma_{t}$ & $\mu_{t}$ & $\sigma_{t}$ \\
\hline $\mathrm{SNR}=32 \mathrm{~dB}$ & 50.0 & 0.0 & 50.0 & 0.0 & 50.0 & 0.0 & 50.0 & 0.0 \\
\hline $16 \mathrm{~dB}$ & 50.2 & 0.4 & 49.4 & 3.9 & 50.2 & 0.4 & 49.4 & 3.9 \\
\hline \multirow[t]{4}{*}{$8 \mathrm{~dB}$} & 49.3 & 0.5 & -80.6 & 412 & 49.3 & 0.5 & -80.6 & 412 \\
\hline & \multicolumn{8}{|c|}{ Step change with different amplitude } \\
\hline & \multicolumn{2}{|c|}{ WCCC } & \multicolumn{2}{|c|}{ WECC } & \multicolumn{2}{|c|}{ WCCC } & \multicolumn{2}{|c|}{ WECC } \\
\hline & $\mu_{t}$ & $\sigma_{t}$ & $\mu_{t}$ & $\sigma_{t}$ & $\mu_{t}$ & $\sigma_{t}$ & $\mu_{t}$ & $\sigma_{t}$ \\
\hline$A=0.25$ & 50.0 & 0.0 & 50.0 & 5.3 & 50.0 & 0.0 & 50.0 & 5.3 \\
\hline 0.5 & 50.2 & 0.4 & 50.5 & 0.7 & 50.2 & 0.4 & 50.5 & 0.7 \\
\hline 2 & 50.2 & 0.4 & 50.2 & 0.4 & 50.2 & 0.4 & 49.9 & 3.4 \\
\hline 4 & 49.7 & 0.5 & 49.7 & 0.5 & 49.7 & 0.5 & 49.4 & 4.0 \\
\hline \multirow[t]{4}{*}{8} & 50.0 & 0.0 & 50.0 & 0.5 & 50.0 & 0.0 & 50.0 & 0.5 \\
\hline & \multicolumn{8}{|c|}{ Exponential decay } \\
\hline & \multicolumn{2}{|c|}{ WCCC } & \multicolumn{2}{|c|}{ WECC } & \multicolumn{2}{|c|}{ WCCC } & \multicolumn{2}{|c|}{ WECC } \\
\hline & $\mu_{t}$ & $\sigma_{t}$ & $\mu_{t}$ & $\sigma_{t}$ & $\mu_{t}$ & $\sigma_{t}$ & $\mu_{t}$ & $\sigma_{t}$ \\
\hline$b=4$ & 50.0 & 0.0 & 50.0 & 0.0 & 50.0 & 0.0 & 50.0 & 0.0 \\
\hline 2 & 50.0 & 0.0 & 50.0 & 0.0 & 50.0 & 0.0 & 50.0 & 0.0 \\
\hline 1 & 50.0 & 0.0 & 50.0 & 0.0 & 50.0 & 0.0 & 50.0 & 0.0 \\
\hline 0.5 & 50.0 & 0.0 & 50.0 & 0.0 & 50.0 & 0.0 & 50.0 & 0.0 \\
\hline \multirow[t]{4}{*}{0.25} & 50.0 & 0.0 & 50.0 & 0.0 & 50.0 & 0.0 & 50.0 & 0.0 \\
\hline & \multicolumn{8}{|c|}{ Linear decay } \\
\hline & \multicolumn{2}{|c|}{ WCCC } & \multicolumn{2}{|c|}{ WECC } & \multicolumn{2}{|c|}{ WCCC } & \multicolumn{2}{|c|}{ WECC } \\
\hline & $\mu_{t}$ & $\sigma_{t}$ & $\mu_{t}$ & $\sigma_{t}$ & $\mu_{t}$ & $\sigma_{t}$ & $\mu_{t}$ & $\sigma_{t}$ \\
\hline$c=4$ & 50.0 & 0.0 & 50.0 & 0.0 & 50.0 & 0.0 & 50.0 & 0.0 \\
\hline 2 & 50.0 & 0.0 & 50.0 & 0.0 & 50.0 & 0.0 & 50.0 & 0.0 \\
\hline 1 & 50.0 & 0.0 & 50.0 & 0.0 & 50.0 & 0.0 & 50.0 & 0.0 \\
\hline 0.5 & 50.0 & 0.0 & 49.8 & 0.4 & 50.0 & 0.0 & 49.8 & 0.4 \\
\hline 0.25 & 50.0 & 0.0 & 50.0 & 0.0 & 50.0 & 0.0 & 50.0 & 0.0 \\
\hline
\end{tabular}

13 of 15

American Institute of Aeronautics and Astronautics 


\section{Conclusions}

The time-of-flight of a propagating shock or detonation wave is an important parameter in experimental studies of such phenomena. Generally, the TOF is determined visually, which introduces a certain amount of arbitariness, especially in quantifying the uncertainty of the estimate. Quantitative methods were proposed previously, utilizing cross-correlation ${ }^{6}$ and cross-spectra. ${ }^{7}$ Haar and Morlet wavelet methods were developed to obtain the TOF estimate by testing them against model functions. This study showed that these wavelets can be applied to shock waves provided that the SNR ratio is good, as is usually encountered in shock tubes. Moreover, both wavelet methods worked very well for detonation waves. One can conclude that wavelet methods work well with spiky profiles. For step discontinuities such as shock waves, the noise level should be low.

\section{Acknowledgement}

This work is partly funded by the National University of Singapore via Research Collaboration Agreement No. TL/AE/2008/01.

\section{A. Wavelet Scales}

The scales used for the wavelet methods can be related to frequency. A pseudo-frequency $F_{a}$ is used to correspond to a given scale $a$ for a wavelet. The relationship used to relate the pseudo-frequency and scale is the following

$$
F_{a}=F_{c} F_{s} / a
$$

where the center frequency of the wavelet $F_{c}$ varies depending on the wavelet function used. The center frequency of the wavelet represents a periodic signal that matches the mother wavelet function. The periodic signal is the best approximation that captures the main wavelet oscillations. ${ }^{18}$ An example of a periodic signal (blue line) approximating a Morlet

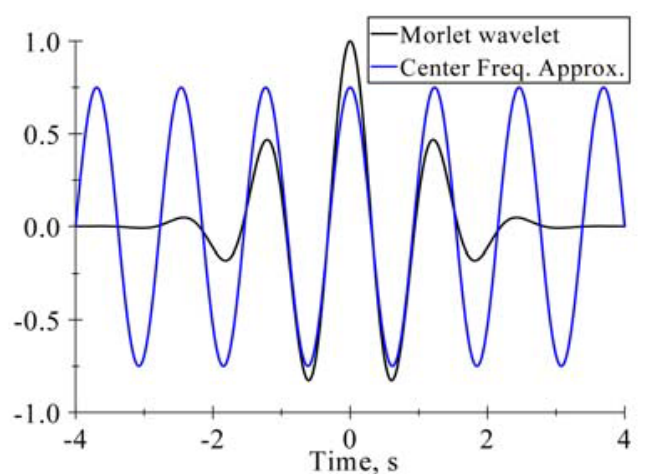

Figure 15. Center frequency approximation of the Morlet wavelet function. wavelet (black line) is provided in Fig. 15. The approximation is very good for the main oscillations. For the smaller oscillations, the approximation drifts from the actual oscillating effect produced by the wavelet function. Since the center frequency of the wavelet is an approximation, the relationship between scale and frequency is not exact. Hence, a pseudo-frequency is introduced that closely approximates a frequency for a given scale. The center frequency for both the Haar and Morlet wavelet functions for this example are 0.9961 and $0.8125 \mathrm{~Hz}$ respectively. The Haar function is a uniform periodic function, so it has nearly a 1:1 ratio for the center frequency approximation. The Morlet wavelet has a center frequency that is less than unity because the period between oscillations is greater than one.

An example of converting a scale to a frequency is provided by the following. For the Morlet wavelet, the center frequency of the wavelet is $0.8125 \mathrm{~Hz}$. The shock tube data were captured at $500 \mathrm{kHz}$. Hence, in this example, the pseudo-frequency for a scale value of 10 for the Morlet wavelet is $40625 \mathrm{~Hz}$.

\section{References}

${ }^{1}$ Ishii, K., Kataoka, H. and Kojima, T., "Initiation and Propagation of Detonation Waves in Combustible High Speed Flows," Proceedings of the Combustion Institute, Vol. 32, 2009, pp. 2323-2330.

14 of 15 
${ }^{2}$ Kontis, K., An, R., Kounadis, D. and Zare-Behtash, H., "Head-On Collision of Shock Wave Induced Vortices with a Cylinder and a Sphere," International Journal of Heat and Fluid Flow, Vol. 29, No. 5, 2008, pp. 1380-1392.

${ }^{3}$ Rakitin, A.E. and Starikovskii, A.Y., "Mechanisms of Deflagration-to-Detonation Transition Under Initiation by HighVoltage Nanosecond Discharges," Combustion and Flame, vol. 155, No. 1-2, 2008, pp. 343-355.

${ }^{4}$ Borgioli, G., Capineri, L., Falorni, P., Matucci, S. and Windsor, C.G., "The Detection of Buried Pipes from Time-of-Flight Radar Data," IEEE Transactions on Geoscience and Remote Sensing, Vol. 46, No. 8, 2008, pp. 2254-2266.

${ }^{5}$ Amorini, F., Anzalone, A., Bassini, R., Boiano, C., Cardella, G., Cavallaro, S., De Filippo, E., Guazzoni, P., La Guidara, E., Lanzanò, G., Pagano, A., Papa, M., Pirrone, S., Politi, G., Porto, F., Riccio, F., Rizzo, F., Russo, S., Russotto, P. and Zetta, L., "Digital Signal Processing for Mass Identification in a $4 \pi$-Detector, Using Time of Flight Measurement," IEEE Transactions on Nuclear Science, Vol. 55, No. 2, 2008, pp. 717-722.

${ }^{6} \mathrm{Lu}$, F.K., Ortiz, A.A., Li, J.-M., Kim, C.H. and Chung, K.-M., "Detection of Shock and Detonation Wave Propagation by Cross Correlation," Mechanical Systems and Signal Processing, Vol. 23, No. 4, 2009, pp. 1098-1111.

${ }^{7}$ Ortiz, A.A. and Lu, F.K., "Detection of Wave Propagation by Nonstationary Cross-Correlation and Cross-Spectral Density Phase," AIAA Paper 2009-2577, 2009,

${ }^{8}$ Bendat, J.S. and Piersol, A.G., Engineering Applications of Correlation and Spectral Analysis, 2nd ed., John Wiley \& Sons, Inc., New York, 1993.

${ }^{9}$ Bendat, J.S. and Piersol, A.G., Random Data Analysis and Measurement Procedures, 3rd ed., John Wiley \& Sons, Inc., New York, 2000

${ }^{10}$ Vetterli, M. and Kovačević, J., Wavelets and Subband Coding, Prentice Hall PTR, Upper Saddle River, New Jersey, 2007; http://waveletsandsubbandcoding.org/

${ }^{11}$ Trapier, S., Deck, S., Duveau, P. and Sagaut, P., "TimeFrequency Analysis and Detection of Supersonic Inlet Buzz," AIAA J., Vol. 45, No. 9, 2007, pp. 2273-2284.

${ }^{12}$ Teolis, A., Computational Signal Processing with Wavelets, Birkhäuser, Boston, Massachusetts, 1998.

${ }^{13}$ Chui, C.K., An Introduction to Wavelets, Academic Press, Boston, Massahusetts, 1992.

${ }^{14}$ Qian, S., Introduction to Time-Frequency and Wavelet Transformations, Prentice Hall PTR, Upper Saddle River, New Jersey, 2002

${ }^{15}$ Addison, P.S., The Illustrated Wavelet Transform Handbook: Introductory Theory and Applications in Science, Engineering, Medicine and Finance, CRC Press, Boca Raton, Florida, 2002.

${ }^{16}$ Tiscareno, M.S., Burns, J.A., Nicholson, P.D., Hedman, M.M. and Porco, C.C., "Cassini Imaging of Saturns Rings II. A Wavelet Technique for Analysis of Density Waves and Other Radial Structure in the Rings," Icarus, Vol. 189, No. 1, 2007, pp. $14-34$.

${ }^{17} \mathrm{Li}$, H. and Nozaki, T., "Applications of Wavelet Cross-Correlation Analysis to a Plane Turbulent Jet," Japan Society of Mechanical Engineers International Journal Series B, Vol. 40, No. 1, 1997, pp. 58-66.

${ }^{18}$ Anon., Wavelet Toolbox ${ }^{\text {TM}}$ : scal2frq, MathWorks, Natick, Massachusetts, 2008, http://www.mathworks.com/. 